\title{
Fingerprint Pore Matching based on Sparse Representation
}

\author{
Feng Liu, Qijun Zhao, Lei Zhang, David Zhang \\ Biometrics Research Centre, Department of Computing, The Hong Kong Polytechnic University \\ Hong Kong, China \\ \{csfliu, csqjzhao,cslzhang,csdzhang\}@comp.polyu.edu.hk
}

\begin{abstract}
This paper proposes an improved direct fingerprint pore matching method. It measures the differences between pores by using the sparse representation technique. The coarse pore correspondences are then established and weighted based on the obtained differences. The false correspondences among them are finally removed by using the weighted RANSAC algorithm. Experimental results have shown that the proposed method can greatly improve the accuracy of existing methods.
\end{abstract}

Keywords-pore matching; sparse representation; weighted RANSAC (WRANSAC)

\section{INTRODUCTION}

Sweat pores and other extended fingerprint features are attracting increasing attention from researchers and practitioners working on automatic fingerprint recognition systems (AFRS) [1]. They have been proven to be very useful for improving the accuracy of existing minutiaebased AFRS [2-7]. In this paper, we will focus on the matching of fingerprint pores.

Roughly, existing pore matching methods can be divided into two categories. Methods in the first category base the pore matching on the alignment estimated by using other features such as minutiae [2, 4-6], whereas those in the second category directly pair pores on fingerprints based on their local features and then refine the pore correspondences based on certain global transformation model [7]. Compared with the first kind of methods, the direct pore matching method has been shown to be more effective [7].

However, the existing direct pore matching method still has the following two shortcomings. First, it uses an intensity-correlation-based method to find coarse pore correspondences in which one pore has only one corresponding counterpart. Second, the classical RANSAC (RANdom SAmple Consensus) algorithm is employed which does not consider the different similarities between different pairs of corresponding pores. The intensity correlation is sensitive to noise, which leads to poor performance on low quality fingerprint images. Moreover, the one-to-one coarse pore correspondences would be highly possible to miss some true correspondences. As shown in Fig. 1, when the local patches have mainly parallel ridges (red circle), there is no guarantee that the true pore correspondences can always obtain higher similarities than the false ones in the coarse matching step. Hence, it is better for us to maintain more than one corresponding pores for one pore during coarse matching. We will further demonstrate this in the experimental results. With respect to the second shortcoming, by assigning each pore correspondence a weight based on the difference between the two pores and using the weighted RANSAC (WRANSAC) algorithm [8], we will be enabled to find out the true pore correspondences more efficiently.

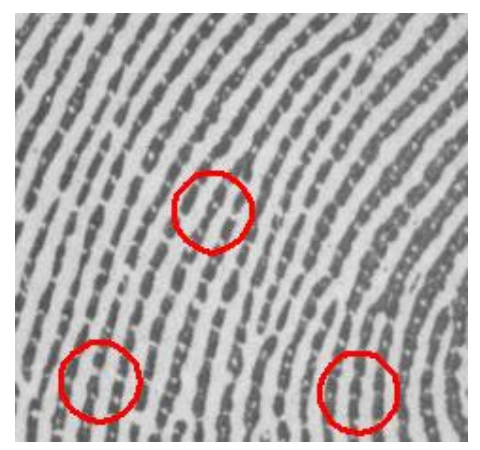

Figure 1. Example local patches consisting of parallel ridges.

This paper thus proposes an improved direct pore matching method. It first establishes one-to-many coarse pore correspondences based on the differences between pores calculated by using the sparse representation (SR) technique which is more robust to noise [9-10]. It then uses WRANSAC to refine the pore correspondences such that the true ones can be obtained more efficiently. Section 2 will introduce in detail the proposed new pore matching method. Section 3 then reports and analyses the experimental results. Section 4 finally concludes the paper.

\section{THE PROPOSED PORE MATCHING}

\section{A. Coarse Pore Correspondences Establishment by SR}

A key issue in establishing the coarse correspondences between pores is to calculate the similarities or differences between the individual pores. Here, we will use the same local descriptors as in [7] for pores so that we can fairly compare the performance of SR-based and correlationbased approaches. The local descriptor $p \in R^{N}$ of a pore as defined in [7] essentially captures the intensity variation in a circular neighborhood to the pore. To calculate the differences between pores, we will use the SR-based rather than correlation-based technique.

Sparse representation has been recently exploited for classification problems [9-10]. Its basic idea is to represent an input sample by linear combination of a set of training 
samples, in which the combination coefficients are restricted to be sparse. It conducts classification based on the assumption that the coefficients corresponding to the samples of the same class have larger absolute values or that the residual of representing the input sample with the samples from the same class is smaller. From the similarity measurement viewpoint, the coefficient associated with each training sample indicates the similarity between this training sample and the input sample, whereas the residual by each class implies the difference between the input sample and the samples in that class. According to the results in [9], the residuals are more robust to noise than the coefficients. Therefore, we measure in this paper the differences between pores by the residuals in sparse representation. In the rest of this subsection, we first introduce the SR-based difference measurement for pores, and then present the proposed method for establishing coarse pore correspondences.

Given an input and a template fingerprints, denote the two sets of pores on them by $P_{1}=\left\{p_{11}, p_{12}, \cdots, p_{1 n}\right\}$ and $P_{2}=\left\{p_{21}, p_{22}, \cdots, p_{2 m}\right\}$, where $n$ and $m$ are the number of pores on the input and template fingerprints, respectively. In order to calculate the difference between each pore in $P_{1}$ and each pore in $P_{2}$, we take each pore in $P_{2}$ as a class, and for each pore in $P_{1}$ we use the linear combination of all the pores in $P_{2}$ to approximate it under the sparse representation constraint. The sparse representation coefficients $x_{j}=\left[x_{j 1}, x_{j 2}, \cdots, x_{j m}\right]^{T}$ ('T denotes transpose) for the $j^{\text {th }}(j=1,2, \ldots, n)$ pore $p_{1 j}$ in $P_{1}$ can be obtained by solving the following $l_{1}$-regularized least squares problem (LSP)

$$
x_{j}=\arg \min \left\|A x_{j}-p_{1 j}\right\|_{2}^{2}+\lambda \sum_{i=1}^{m} x_{j i} .
$$

where $A$ is the basis matrix whose columns are the local descriptors of the pores in $P_{2}$, and $\lambda$ is a free parameter to balance the least squares error of representation and the sparsity of the coefficients. The solution to the above optimization problem can be found in [11].

Based on the obtained sparse representation coefficients, we can calculate the difference between the pore $p_{1 j}$ and the $i^{\text {th }}(i=1,2, \ldots, m)$ pore $p_{2 i}$ in $P_{2}$ as follows

$$
d_{j i}=\left\|p_{2 i} x_{j i}-p_{1 j}\right\|_{2}^{2} \text {. }
$$

After processing all the pores in $P_{1}$, we get the differences of all the $(n \times m)$ pairs of pores between the two fingerprints. Among all these pairs, we choose some as the coarse pore correspondences so that only the pairs with relatively small differences are poured into the subsequent refinement step. This is done as follows.

For each pore in $P_{1}$, the minimum value of the differences between it and the $m$ pores in $P_{2}$ is calculated, which results in $n$ minimum differences. The average of these minimum differences is then calculated. Finally, the pairs of pores whose differences are smaller than the obtained average difference are selected as the coarse pore correspondences. In this way, one pore in $P_{1}$ is allowed to have more than one corresponding pores in $P_{2}$ at this coarse matching step.

\section{B. Pore Correspondences Refinement by WRANSAC}

Because coarse pore correspondences are established based on merely local features, there are usually some false ones among them. The goal of this refinement step is thus to eliminate these false pore correspondences from the obtained coarse pore correspondences. The difference between the method we use here and the method in [7] is that when applying RANSAC, we choose pore correspondences according to the differences between the pores in the correspondences such that the pore correspondences with smaller differences will be chosen with higher probability than those with larger differences. That is we employ the WRANSAC algorithm [8] to refine pore correspondences.

More specifically, we assign to each coarse pore correspondence with a sampling probability (i.e. weight) based on the difference between the two pores in the correspondence. Let $d_{\max }$ be the maximum difference between all pores on the two fingerprints, i.e. $d_{\max }=\max \left\{d_{j i} \mid j=1,2, \cdots, n ; i=1,2, \cdots, m\right\}$. The weight $w$ to the pore correspondence $\left\{p_{1 j}, p_{2 i}\right\}$ is then calculated as follows:

$$
w=1-\frac{d_{j i}}{d_{\max }} .
$$

With the above defined weights, the true pore correspondences with smaller differences can be more quickly found. Meanwhile, the true pore correspondences with relatively large differences (e.g. the ones in one-tomany correspondences which may be due to nondiscriminative or noisy neighbouring patches of the pores) still have chance to be selected. As a result, the proposed method here can find the true pore correspondences not only more efficiently but also more effectively.

\section{EXPERIMENTS AND ANALYSIS}

\section{A. Databases and Protocols}

Two databases of high resolution fingerprint images with resolution $\sim 1200 \mathrm{dpi}$ were used to evaluate the performance of the proposed method. The first database, denoted as DBI, is the same database as the one used in [7], which contains 1,480 fingerprint images from 148 fingers (five images collected for each finger in each of two sessions separated by about two weeks). The images in DBI have a spatial size of 320 pixels by 240 pixels. The fingerprint images in the second database (denoted as DBII) were collected in the same way but with a larger image size, 640 pixels by 480 pixels.

The pores on all the fingerprint images were extracted by using an improved version of the algorithm in [12] and the minutiae were extracted and matched by using the 
method in [13] (denoted by MINU). We first compared the proposed method (denoted by SRDP) with the existing direct pore matching method [7] (denoted by DP) in terms of their pore correspondences establishment performance, and then compared the fingerprint recognition accuracy of them and the minutiae and ICP (Iterative Closest Points) based method [5-6] (denoted by MICPP).

\section{B. Pore Correspondences Establishment Performance}

Fig. 2 gives an example genuine pair of fingerprint images from DBI to illustrate the effectiveness of SR-based one-to-many coarse pore correspondences establishment and WRANSAC-based pore correspondences refinement. The first 50 coarse pore correspondences obtained by the correlation-based method is shown in Fig. 2(a), among which 11 pairs are false correspondences as highlighted by red color. Fig. 2(b) shows the first 50 coarse pore correspondences acquired by SR-based method, which include only 5 false ones (also marked in red color). Moreover, there are one-to-many coarse pore correspondences in the coarse pore correspondences established by the proposed method, which can be clearly seen in Fig. 2(c) (purple lines). The correct pore correspondences among these one-to-many coarse corresponding pore pairs are finally picked out by the WRANSAC method, as shown in Fig. 2(d), denoted by yellow line. The results shown in Fig. 2 fully demonstrate the effectiveness of our proposed SRDP method.

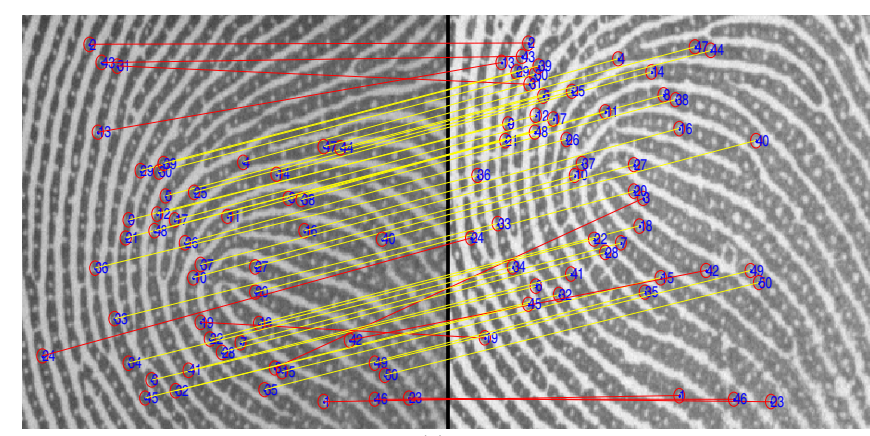

(a)

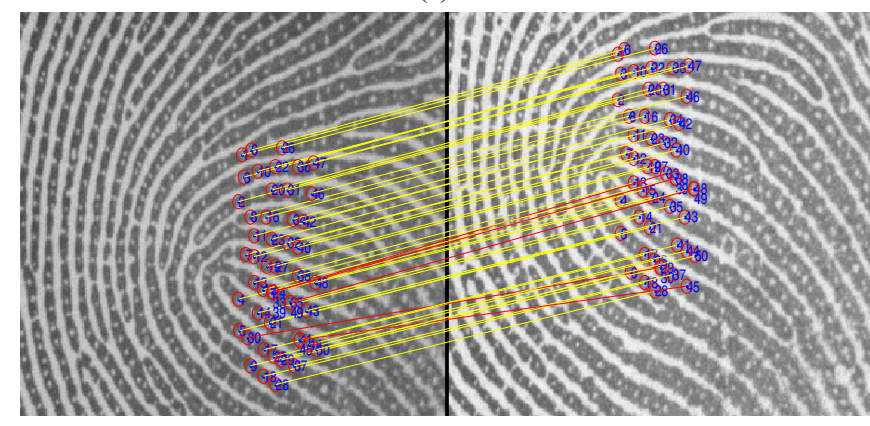

(b)

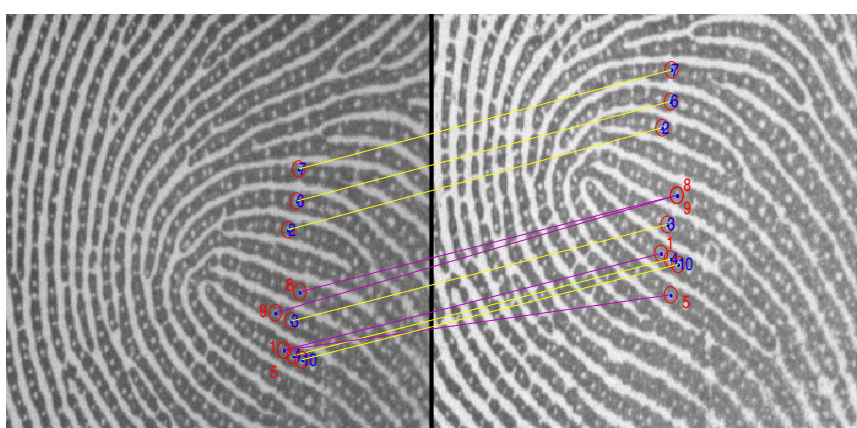

(c)

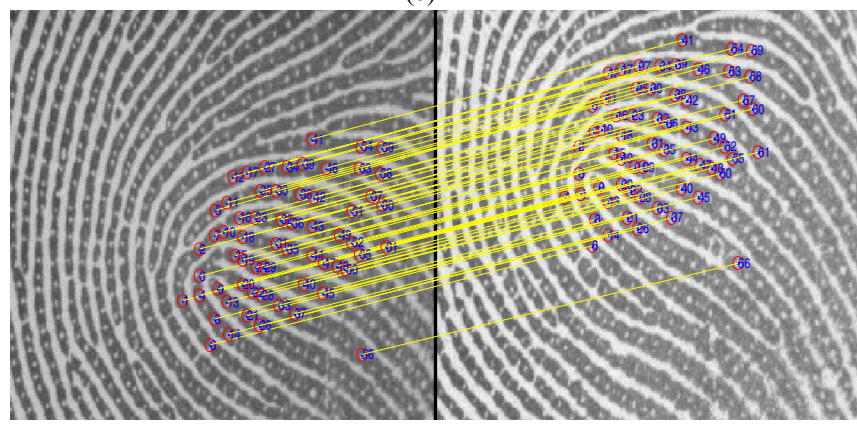

(d)

Figure 2. The first 50 coarse pore correspondences obtained by (a) correlation-based and (b) SR-based methods. (c) Examples of one-to-many coarse pore correspondences obtained by SR-based method. (d) The final pore correspondences after using WRANSAC to refine the coarse pore correspondences established by SR-based method.

\section{Fingerprint Recognition Accuracy}

In order to evaluate the fingerprint recognition accuracy by using the proposed method and its counterparts, we conducted the following matches for each method on both DBI and DBII. 1) Genuine matches: each of the fingerprint images in the second session was matched with all the fingerprint images of the same finger in the first session, resulting in 3,700 genuine match scores. 2) Imposter matches: the first fingerprint image of each finger in the second session was matched with the first fingerprint images of all the other fingers in the first session, resulting in 21,756 imposter match scores. Note that the pore match scores in our experiments were defined as the number of pairs of finally matched pores on fingerprints, which is different from the one used in [7]. Based on the obtained match scores, the equal error rates (EER) were calculated for each method. The fusion between pores and minutiae has been also considered, and the weighted sum rule as in [7] was employed to integrate the pore match scores and minutiae match scores. Different weights from 0.1 to 0.9 with an interval as 0.1 were tested and the best EER was reported here. For simplicity, we denote the fusion of the match scores by MINU and DP as MINU_DP, and the fusion of the match scores by MINU and SRDP as MINU_SRDP. The EERs obtained by the methods mentioned above are shown in Table I. 
Table I. EER of different methods.

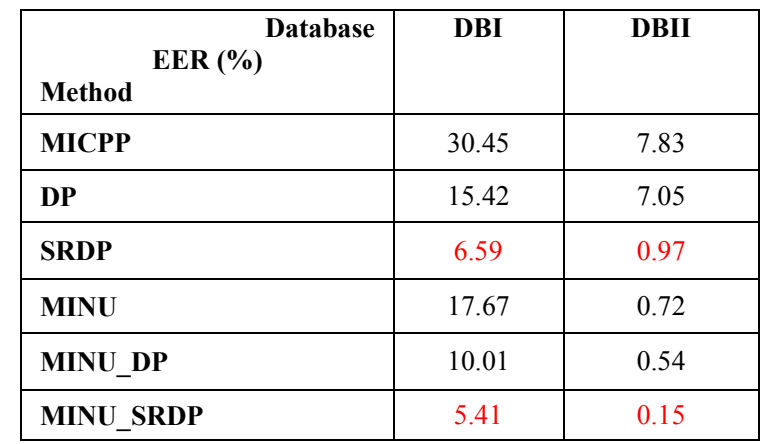

From the experimental results in Table I, we can see that the proposed method outperforms both MICPP and DP by decreasing the EER by one order of magnitude on both DBI and DBII. When fusing the match scores of minutiae and pores, the proposed method again overwhelms the existing DP method. It can be clearly seen from Table I that MINU_SRDP improves the accuracy of MINU_DP by about $46 \%$ and $72 \%$ on DBI and DBII respectively.

We believe that the improvement achieved by the proposed method owes to the following two factors. First, the sparse representation based method can better handle the noise, which has also been demonstrated in [9]. Second, the one-to-many coarse pore correspondences establishment scheme together with the WRANSAC based refinement make it more effective to find the correct correspondences between pores on fingerprints. However, the high computational complexity caused by the large number of pores on fingerprints is still a problem we have not solved as in [7]. Firstly align two fingerprints to estimate the overlapping area between them before pore matching will somewhat ease the computational complexity problem by reducing many invalid pores. Selecting distinctive pores from the original large pore set is another way to reduce the computational complexity. Our future work will mainly focus on the selection of distinctive pores for matching.

\section{CONCLUSION}

This paper introduced a novel direct pore matching method based on sparse representation and the WRANSAC algorithm. Different from the existing direct pore matching method, it measures the differences between pores based on the residuals of sparse representation, establishes one-tomany pore correspondences in the coarse matching step, and takes the differences between pores as the weights of the correspondences in the refinement step. The experimental results demonstrate that the proposed method can more effectively establish pore correspondences and greatly improve the fingerprint recognition accuracy by reducing the EER by one order of magnitude when using only pores and by up to $70 \%$ when fusing pores and minutiae.

\section{ACKNOWLEDGMENT}

This research is partially supported by the HK PolyU internal research grant G-YH54.

\section{REFERENCES}

[1] CDEFFS, Data Format for the Interchange of Extended Fingerprint and Palmprint Features, Version 0.4, 2009.

[2] J. Stosz and L. Alyea, "Automated system for fingerprint authentication using pores and ridge structure," in Proc. SPIE, 1994, pp. 210-223.

[3] A. Roddy and J. Stosz, "Fingerprint features - statistical analysis and system performance estimates," Proc. IEEE, vol. 85, no. 9, pp. 1390-1421, Sep. 1997.

[4] K. Kryszczuk, P. Morier, and A. Drygajlo. "Study of the distinctiveness of the level 2 and level 3 features in fragmentary fingerprint comparison," Proc. Biometrics Authentication, ECCV 2004 International workshop, 2004, pp.124-133.

[5] A. Jain, Y. Chen, and M. Demirkus, "Pores and ridges: Fingerprint matching using level 3 features," Proc.18th International Conference on Pattern Recognition, 2006, pp. 477-480.

[6] A. Jain, Y. Chen, and M. Demirkus, "Pores and ridges: Highresolution fingerprint matching using level-3 features," IEEE Trans. Pattern Analysis and Machine Intelligence, vol. 29, no. 1, pp. 15-27, Jan. 2007.

[7] Q. Zhao, L. Zhang, D. Zhang, and N. Luo, "Direct pore matching for fingerprint recognition," ICB'09, 2009, pp. 597 606.

[8] D. Zhang, W. Wang, Q. Huang, S. Jiang, and W. Gao, "Matching images more efficiently with local descriptors," ICPR'08, 2008.

[9] J. Wright, A. Y. Yang, A. Ganesh, S. Sastry, and Y. Ma, "Robust face recognition via sparse representation," IEEE Trans. Pattern Analysis and Machine Intelligence, vol. 31, no. 2, pp. 210-227, Feb. 2009.

[10] S. Yan and H. Wang, "Semi-supervised learning by sparse representation," SDM, pp. 792-801, 2009.

[11] K. Koh, S. Kim, S. Boyd , and D. Gorinevsky, "An interiorpoint method for large-scale $l_{1}$-regularized least squares," IEEE Journal on Selected Topics in Signal Processing, vol. 1, no. 4, pp. 606-617, 2007.

[12] Q. Zhao, L. Zhang, D. Zhang, N. Luo, and J. Bao, “Adaptive pore model for fingerprint pore extraction," ICPR'08, 2008.

[13] J. Feng, Combining minutiae descriptors for fingerprint matching," Pattern Recognition, vol. 41, pp. 342-352, 2008. 